\title{
L'ATTUALE FASE DI ATTUAZIONE DELLA CONVENZIONE UNESCO 1970 SULLA PROTEZIONE DEI BENI CULTURALI. GLI ESITI DI UN SEMINARIO INTERNAZIONALE (MEXICO SEMINAR 2013)
}

\author{
Nota del s.c. ANTONIO GAMBARO (*)
}

(Adunanza del 23 maggio 2013)

\begin{abstract}
SunTO. - La Nota riferisce circa gli esiti del Mexico Seminar 2013 indetto dall' International Association of Legal Sciences (una organizzazione affiliata all'UNESCO) per fare il punto sullo stato di attuazione della Convenzione UNESCO del 1970 On the Means of Probibiting and Preventing the Illicit Import, Export and Transfer of Ownership of Cultural Property. Il seminario messicano, cui hanno partecipato giuristi, diplomatici ed esperti da varie parti del mondo, ha concentrato la propria attenzione sui beni archeologici ponendo in luce le ragioni di carattere giuridico che ostacolano le azioni di rivendicazione intraprese dagli Stati dal cui territorio tali beni sono stati esportati. Ragioni non del tutto superate dalla UNIDROIT Convention on Stolen or Illegally Exported Cultural Objects of 24 June 1995 e dalla UNESCO-UNIDROIT del 2011, Model Provisions on State Ownership of Undiscovered Cultural Objects.
\end{abstract}

$* * *$

ABSTRACT. - The Note reports about the Mexico Seminar 2013 organized by the International Association of Legal Sciences (International association affiliated with UNESCO). The Seminar has focused its attention on the state of implementation of the 1970 UNESCO Convention on the Means of Prohibiting and Preventing the Illicit Import, Export and Transfer of Ownership of Cultural Property, followed by the UNIDROIT Convention on Stolen or Illegally Exported Cultural Objects of 24 June

(*) Università degli Studi di Milano, Italy.

E-mail: antonio.gambaro@unimi.it 
1995 and the 2011 UNESCO-UNIDROIT Model Provisions on State Ownership of Undiscovered Cultural Objects. In the Mexico seminar jurists and officials of various parts of the world have pointed out the weakness of the legal framework created by the conventions and therefore the difficulties in the exercise of restitutionary remedies by the States from which a cultural object has been removed.

Ho chiesto al Presidente di poter riferire a questa Accademia sugli esiti di un seminario internazionale cui ho partecipato, perché ritengo che il tema del seminario, la protezione internazionale dei beni archeologici, possa interessare non pochi tra i soci, ed anche perché sul tema della circolazione internazionale dei beni culturali si riscontra una preoccupante dose di disinformazione.

Il problema della circolazione internazionale dei beni archeologici è uno spicchio del tema della circolazione dei beni culturali e perciò si deve anzitutto registrare il mutamento di paradigma avvenuto nella seconda metà del secolo scorso.

Ad iniziare dalla UNESCO Convention del 1970 On the Means of Probibiting and Preventing the Illicit Import, Export and Transfer of Ownership of Cultural Property, si è affermato nel mondo un principio innovativo che inclina verso la preservazione dei beni culturali presso la comunità che li ha prodotti. Tale principio prevede molte attenuazioni: non riguarda i beni culturali di nuova produzione e non riguarda i beni culturali che hanno circolato nel passato perché la Convenzione, come le altre che sono seguite, non ha effetto retroattivo. Tuttavia il principio della preservazione rimane il caposaldo del nuovo modello di disciplina della circolazione internazionale dei beni culturali.

Quarantatre anni dopo, si può dire che la Convezione del 1970 è stata un grande successo posto che è stata ratificata da 123 Stati membri dell'UNESCO.

Tuttavia la Convenzione del 1970 è appositamente lacunosissima in tema di regole giuridiche e ciò svela che il suo intento è stato quello di fissare un principio di civiltà giuridica internazionale, senza pretendere di renderlo immediatamente operativo ${ }^{1}$.

1 Cfr. L.V. Prott [Lyndel V.], Problems of Private International Law for the Protection of the Cultural Heritage, in Academie de la Haie de droit Intern. Recueil des Cours, 1989, V, p. 219; S.A. Williams [Sharon A.], The International and National Protection of Movable Cultural Property. A Comparative Study, New York- Dobbs Ferry, 1978. 
Il risultato è stato che, nei primi trent'anni di vigenza, quasi sempre gli Stati che rivendicavano il possesso di beni culturali, specie archeologici, che siano stati illegalmente trasportati all'estero non sono riusciti a riottenerli ${ }^{2}$.

Inizialmente si è ritenuto che il problema fosse l'assenza di regole giuridiche precise e quindi si è tentato di colmare la lacuna mediante la UNIDROIT Convention on Stolen or Illegally Exported Cultural Objects of 24 June $1995^{3}$ e le UNESCO-UNIDROIT Model Provisions on State Ownership of Undiscovered Cultural Objects 2011.

La convenzione UNIDROIT 1995 prevede in effetti regole giuridiche piuttosto precise che imprimono ai beni culturali un regime di circolazione internazionale assai diverso rispetto al regime di circolazione ordinario dei beni mobili e quindi consentono di distinguere tra circolazione lecita e circolazione illecita di beni culturali. Ma la Convenzione UNIDROIT ha avuto un successo molto limitato; sono solo 33 gli Stati firmatari e mancano tutti i più importanti Stati di recezione. La UNESCO-UNIDROIT Model Provisions on State Ownership of Undiscovered Cultural Objects 2011 è una soft Law e quindi priva di efficacia giuridica immediata.

Come è emerso in modo assai netto a Mexico le speranze che la UNIDROIT Convention 1995 possa essere ratificata dagli Stati di maggior rilevanza nel commercio internazionale stanno svanendo. Da ciò l'idea di chiedere lumi alle associazioni scientifiche internazionali che all'UNESCO sono collegate. Essenzialmente, nel caso, alla IALS' che ha promosso il seminario internazionale svoltosi a Mexico City nel marzo 2013, sul cui svolgimento intendo brevemente riferire.

$\mathrm{Va}$ anzitutto posto in luce come il seminario messicano abbia registrato presenze significative sia a livello accademico che a livello di rappresentati dei governi e ciò ha consentito uno scambio informato di

2 Cfr. U.S. v. Mc Clain, 593 F2d 658 (1979).

3 Cfr per tutti M. Schneider, UNIDROIT Convention on Stolen or Illegally Exported Cultural Objects: Explanatory Report, in Unif. L. Rev. 2001. 476

4 Cfr per tutti: M. Frigo, Model Provisions on State Ownership of Undiscovered Cultural Objects - Introduction, Unif. L. Rev. 2011, 1024.

5 L'International Association of Legal Science - Association Internationale des Sciences juridiques è stata istituita nel 1950 ed opera sotto gli auspici dell'UNESCO e dell'International Social Science Council che ha contribuito a fondare; ha tra suoi membri esclusivamente associazioni nazionali che si dedicano allo sviluppo degli studi giuridici sul piano transnazionale. 
idee ed esperienze. Tuttavia l'obiettivo del seminario era capire la radice dei problemi.

Tra i varî problemi ve ne sono alcuni che qui non menziono perché impingono sulle technicalities del diritto internazionale.

Il problema essenziale per capire che cosa non ha funzionato sta nel meccanismo delle restituzioni.

Non è pensabile, anche se qualcuno lo ha pensato, di agire in via amministrativa. Per ottenere la restituzione di un bene che è in possesso di altri occorre il consenso del possessore, oppure si deve agire in via giudiziale. Questa banale osservazione aiuta a ricordare che la Convenzione UNESCO 1970 è stata concepita in un'atmosfera culturale in cui una corposa corrente di pensiero considerava che la proprietà privata fosse una istituzione destinata a scomparire in un tempo sperabilmente breve; però la Convenzione si è trovata a vivere in un mondo ove la tutela dei property rights è divenuta il cardine dell'ordine giuridico globale.

Da ciò discende che lo Stato, o il soggetto illecitamente spogliato di un suo bene culturale che sia stato trasportato all'estero, deve assumere avanti la giurisdizione dello Stato estero la posizione dell'attore che esercita un'azione di rivendicazione. Deve quindi provare che quell'oggetto è una sua proprietà. Vi è da osservare che in materia di azioni reipersecutorie i diversi sistemi giuridici hanno prodotto un complesso mosaico di soluzioni rispetto alle quali non è facile orientarsi; tuttavia simile diversità non è rilevante ai fini del discorso qui condotto perché sotto il profilo operazionale la meccanica processuale fondamentale è identica. Chi rivendica il possesso di un oggetto pretendendo che sia di sua proprietà deve provare il titolo che gli conferisce il diritto di proprietà. Apparentemente le difficoltà maggiori insorgono qualora il diritto dello Stato in cui si agisce si adegui al principio per cui en fait de meubles possession vaut titre, e ciò ha indotto UNIDROIT ad introdur-

6 Cfr in breve: A-F. Zattera, La dimension constitutionelle et européenne du droit de propriété, Paris, LGDJ, 2001; A. Candian, I diritti fondamentali in Europa, in AA.VV., I diritti fondamentali in Europa, Milano, Giuffré, 2002, p. 63; S. Pavegeau, Le droit de propriété dans les jurisprudences suprêmes françaises, européennes et internationales, Paris LGDJ, 2006; G.S. Alexander, The Global Debate over Constitutional Property. Lessons for American Takings Jurisprudence, Chicago Univ.Press., 2006; S. Praduroux, The European Dimension of Property as a Fundamental Right and its Impact on French and Italian Law, Helsinki, 2012. 
re regole convenzionali che escludono questa presunzione in tema di beni culturali. Ma rovesciare la presunzione di legittimità del possesso, come ha tentato di fare la Convenzione UNIDROIT, è una operazione piuttosto assurda perché, se è pensabile privare il possessore attuale del vantaggio discendente da una presunzione di legittimità del possesso, rimane logicamente impossibile assistere il rivendicante in quanto tale con una presunzione di titolarità della proprietà. Pertanto costui dovrà sempre addurre e provare una serie di fatti da cui ricavare una presunzione di titolarità a suo favore.

Pertanto i punti fermi da cui partire si possono riassumere così:

a) non è possibile procedere ad una restituzione di beni se non con il consenso del possessore oppure mediante la decisione del Tribunale del luogo ove il bene si trova (ne è riprova il fallimento dell' Intergovernmental Committee for Promoting the Return of Cultural Property to its Countries of Origin or its Restitution in Case of Illicit Appropriation, creato in seno all'UNESCO; quest'organo, che avrebbe dovuto sovraintendere a restituzioni agendo per via sostanzialmente amministrativa, ha prodotto esiti deludenti: solo 12 casi di restituzione in trent'anni di cui 4 per decisione giudiziaria e 2 per accordo tra le parti);

b) la decisione del Tribunale, o della Corte, che accoglie o respinge una domanda di rivendicazione è sempre il risultato di una complessa interazione tra regole (legali e consuetudinarie) e mentalità giuridiche; è ingenuo pensare che basti modificare la regola legale per ottenere una decisione conforme alle intenzioni del legislatore; ciò significherebbe credere che i giudici siano solo la bocca inanimata della legge;

c) non è possibile escludere che beni culturali possano essere oggetto di proprietà privata e quindi escludere che al loro titolare apparente si applichino le garanzie che gli ordinamenti normalmente apprestano a favore del diritto di proprietà.

Altre difficoltà che sono state evidenziate in passato si sono dimostrate, all'analisi svoltasi nel Seminario messicano, essere più teoriche che reali.

Così in passato si è osservato che la concretizzazione dei principî espressi nella convenzione UNESCO del 1970 richiede che il regime di circolazione internazionale dei beni culturali si differenzi dal regime di circolazione normale dei beni mobili; ma ciò è possibile solo se si è dotati di una definizione precisa di bene culturale, condizione che la 
Convenzione UNESCO soddisfa solo in piccola parte, altrimenti si grava il rivendicante dell'onere di dimostrare anzitutto che l'oggetto rivendicato rientra nella categoria dei beni culturali ed una volta assolto questo onere di provare che egli ha un titolo migliore del possessore attuale. In definitiva questa critica assume che la vaghezza della nozione di bene culturale aggrava e non migliora la posizione del rivendicante. Inoltre si è osservato che l'ampiezza della nozione di bene culturale che si legge nella Convenzione UNESCO 1970 crea antinomie con altre convenzioni internazionali. Ad esempio l'art. XX(f) del GATT, concluso in seno al WTO, prevede che gli Stati possono sottrarsi agli obblighi di non ostacolare il libero commercio limitatamente all'adozione di regole legali che siano imposed for the protection of national treasures of artistic, bistoric or archaeological value; così individuando una categoria di beni assai più ristretta di quella di "bene culturale" come definita nella Convenzione UNESCO 1970.

Il Seminario messicano è servito a mettere in luce come questa osservazione, in sé piuttosto logica, a livello operativo è irrilevante. Infatti si deve osservare come la necessità di agire in rivendicazione avanti un tribunale straniero comporti un effetto-soglia.

L'esperienza dimostra infatti che le azioni di rivendicazione transnazionali riguardano solo oggetti di notevole valore, che sono oggetti unici e la cui origine ed i cui caratteri sono ben riconoscibili. I dubbi di attribuzione sono casi marginali e perciò trascurabili nel nostro discorso. Qualunque sia il catalogo dei beni culturali, l'effetto-soglia che si collega alla necessità di agire avanti un tribunale estero affrontando i rischi ed i costi di una azione giudiziaria fa sì che le azioni di recupero siano limitate ad oggetti di eccezionale valore storico-culturale.

Ciò posto, si rileva che le iniziative tendenti ad ottenere la restituzione di beni culturali ed in particolare di beni archeologici coinvolgono essenzialmente problemi di prova e pertanto problemi che intersecano anche le mentalità giuridiche dei giudicanti.

A questo riguardo a Mexico sono emerse luci ed ombre.

Negli ultimi anni si è registrato un netto incremento di casi risolti a favore del rivendicante. Vi è stato anche un certo numero di repressioni penali a carico di mercanti di oggetti archeologici illecitamente esportati dai paesi di origine. Quest'ultimo fenomeno è collegato ad un mutamento di mentalità di giudici e pubbliche autorità che è particolarmente riscontrabile negli Stati Uniti, sino a qui il più grande mercato di destinazione degli oggetti aventi valore archeologico. Traffici di beni 
archeologici attuali sono combattuti con un certo vigore, anche per le connessione tra questo tipo di traffico ed altri tipi di traffico illecito come droga ed armi nonché a cagione del sospetto che esistano connessioni significative tra i soggetti implicati tra questo tipo di traffici ed attività terroristiche.

Ciò ha rafforzato la posizione del rivendicante in fase di negoziazione ante judicium. Con esisti positivi specie per lo Stato italiano che ha saputo sfruttare il momento favorevole per concludere accordi di restituzione, che sono sostanzialmente accordi transattivi ${ }^{\top}$. Come in ogni transazione le parti coinvolte nella disputa attorno alla proprietà di un bene archeologico si accordano facendosi reciproche concessioni e pertanto gli accordi conclusi dallo Stato italiano prevedono, accanto alla restituzione del bene archeologico illecitamente esportato, prestiti di lungo termine, la creazione di diritti di comproprietà, oppure scambi di oggetti, oppure ancora progetti di ricerca comuni o esibizioni congiunte volte ad esaltare il valore di un dato bene archeologico. Debbo riferire che simili soluzioni consensuali delle dispute, chiamate orami nel gergo di settore Italian Deal, suscitano interesse ed apprezzamento, perché foriere di risultati restitutori che il sistema attuale non consentirebbe di raggiungere.

Soprattutto, però, si è registrato che sta mutando il codice di condotta dei dealers di opere antiche. Dal classico atteggiamento don't ask, don't tell, circa la provenienza degli oggetti d'arte si è passati a codificare il principio per cui ci si deve accertare della origine lecita. Codici di autodisciplina sono stati adottati su tutte le piazze più importanti. Eguale vicenda riguarda i curatori dei musei.

Un mutamento di mentalità è quindi in corso. Tale fenomeno si svolge lentamente come avviene a tutti i mutamenti di mentalità, ma la direzione del mutamento è ben percepibile.

Per accelerare questo mutamento a Mexico si sono avanzate alcune idee. Molti ritengono che il regime giuridico più adatto ai beni archeologici sia la State Ownership, ma non esiste universale consenso

7 E' anche da ricordare che posizioni di forza negoziale nascono dalla capacità di individuare l'origine precisa di un bene archeologico mediante lo svolgimento di adeguate indagini di polizia. Sotto questo profilo nessun paese è in grado di usufruire di un apparato di elevato livello come quello messo in campo dal Comando Carabinieri Tutela Patrimonio Culturale. 
su questa scelta ed ancor meno sul fatto che la State Ownership debba essere quella del paese di provenienzå

Quest'ultimo punto di arrivo non è affatto previsto dalla Convenzione UNESCO 1970, non è fondabile razionalmente ed è inutile illudersi del contrario'. I beni culturali e quindi anche quelli di valore archeologico hanno sempre circolato; all'epoca in cui alcuni beni archeologici sono stati prodotti, molti Stati neppure esistevano. Pertanto fare riferimento allo Stato di provenienza significa adottare un criterio ambiguo che in quanto tale è del tutto inadatto a risolvere le dispute circa il titolo di proprietà ${ }^{10}$.

Si può quindi suggerire di fondare il principio dell'appartenenza necessaria di certi beni sulla relazione esistente tra una comunità che ritiene tale bene importante alla preservazione del proprio cultural heritage e l'identità culturale che tale comunità si è data. La situazione di appartenenza deve quindi essere pensata in relazione ad una comunità umana attuale che tuttavia abbia titolo per rivendicare la connessione più significativa con ciò che quell'oggetto testimonia. Ne discende che il rapporto tra caratteri del bene ed appartenenza deve essere fondato su un rapporto di connessione culturale, piuttosto che su una connessione di carattere puramente geografico-politico. Infatti, se accettiamo l'idea che un bene culturale è tale perché è dotato di un valore simbolico, dobbiamo svolgere i passaggi conseguenti. Assegnare ai beni culturali un valore simbolico significa riconoscere che i beni culturali sono fonte di messaggi rivolti a chi possa contemplarli o, comunque, fruirne, e perciò essi sono connotati da una valenza necessariamente collettiva in quanto connessi con la cultura di una collettività. Ora, i beni archeologici sono per definizione latori di un messaggio che viene dalla storia remota. Istituire un collegamento meccanico tra tali beni e gli Stati che attualmente governano i territori in cui sono stati prodotti è una opera-

8 Cfr. S.S. Fish (ed.) National Approaches to the Governance of Historical Heritage over Time, Oxford, 2008.

9 Cfr. J.M. Sax, Heritage Preservation as a Public Duty: The Abbé Grégoire and the Origins of an Idea, 88 Mich.L. Rev. 1142(1990); M. Graziadei, Beni culturali (circolazione dei), in Enciclopedia del Diritto- Annali, II, t.2, 91 (2009).

10 Cfr. P. Gerstenblith, Schultz and Barakat: Universal Recognition of National Ownership of Antiquities, 14 Art Antiquity \& L., 21 (2009); K.D. Vitale, The War on Antiquities: United States law and Foreign Cultural property, 84 Notre Dame L. Rev. 1835 (2009). 
zione priva di basi ragionevoli. Chi rivendica un bene archeologico deve dimostrare almeno una certa passione per la propria storia; solo così si può fondare una pretesa rivolta alla preservazione del proprio patrimonio archeologico. Non sempre è il caso. Maria Kouroupas ha indicato esempi di Stati che rivendicano negli USA beni culturali che però non sono protetti come tali nel paese rivendicante.

Pertanto non ci si può illudere di poter fondare una pretesa alla restituzione di beni culturali importanti solo su norme internazionali; occorre anche poter dimostrare passione per il proprio passato ed una coerente serie di azioni giuridiche e culturali al riguardo. 